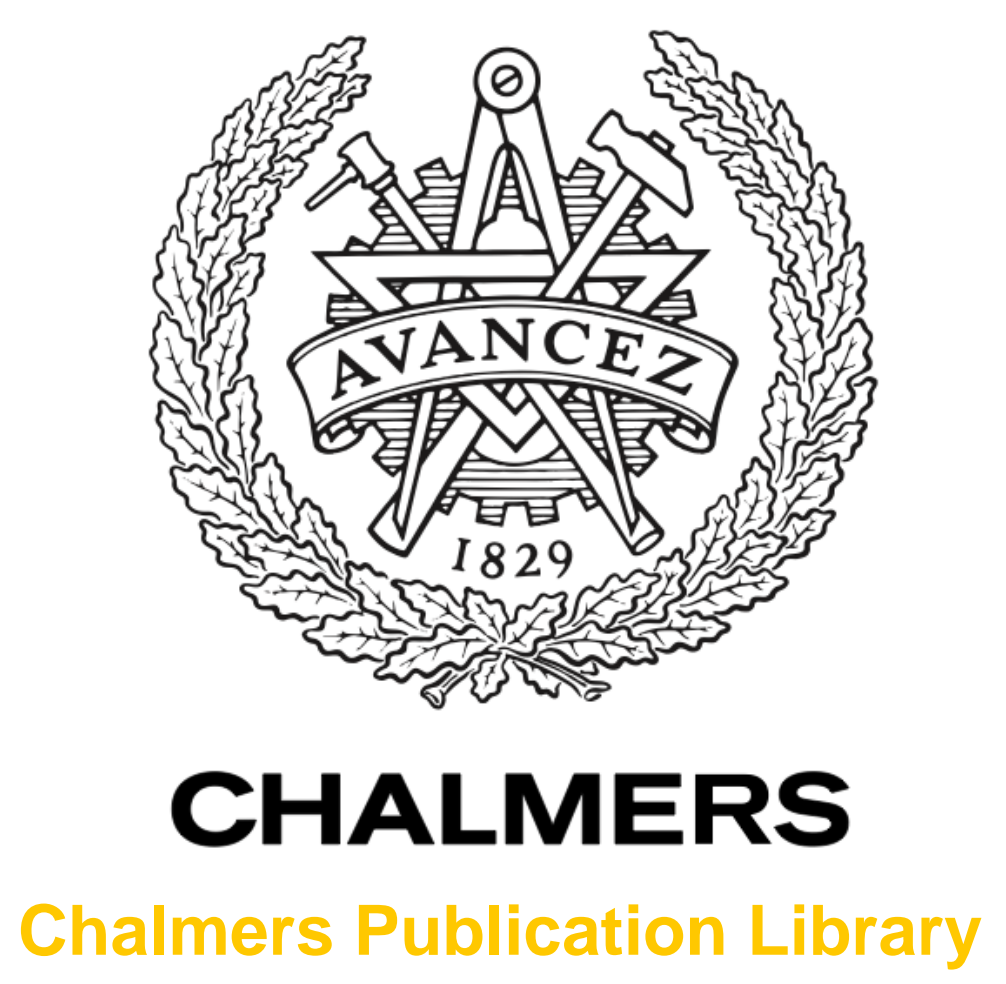

\title{
Coupling effects on richness threshold of multi-port antennas in multipath environments
}

This document has been downloaded from Chalmers Publication Library $(\mathrm{CPL})$. It is the author's version of a work that was accepted for publication in:

15th International Symposium on Antenna Technology and Applied Electromagnetics, ANTEM 2012, Toulouse, 25-28 June 2012

Citation for the published paper:

Jamaly, N. ; Derneryd, A. (2012) "Coupling effects on richness threshold of multi-port antennas in multipath environments". 15th International Symposium on Antenna Technology and Applied Electromagnetics, ANTEM 2012, Toulouse, 25-28 June 2012

http://dx.doi.org/10.1109/ANTEM.2012.6262383

Downloaded from: http://publications.lib.chalmers.se/publication/164214

Notice: Changes introduced as a result of publishing processes such as copy-editing and formatting may not be reflected in this document. For a definitive version of this work, please refer to the published source. Please note that access to the published version might require a subscription. 


\section{Coupling Effects on Richness Threshold of Multi-port Antennas in Multipath Environments}

\author{
Nima Jamaly \\ Chalmers University of Technology \\ Gothenburg, Sweden 41296 \\ Email: jamaly@chalmers.se
}

\author{
Anders Derneryd \\ Ericsson Research, Ericsson AB \\ Gothenburg, Sweden 41756 \\ Email: anders.derneryd@ericsson.com
}

\begin{abstract}
This paper investigates convergence in diversity gain of multi-port antennas in non-rich multipath environments. It is shown that diversity gain convergence is faster in the presence of strong coupling. In this situation, richness threshold is also smaller. Furthermore, by linking convergence in diversity gain to convergence in antenna ports' correlations, the role of pattern diversity caused by coupling is highlighted. A range of variation for correlations is defined whose upper bound is used to quantify pattern diversity. The lower bound for this range is the corresponding spatial correlation in a rich multipath environment.
\end{abstract}

\section{INTRODUCTION}

For decades, the main concern of multi-port antenna designers was to design antennas of negligible correlations but high radiation efficiencies [1]. The concept of mean effective gain has been of further interests, which includes the effects of nonuniform angle of arrival in radiation efficiency of the antennas [2]. But, in most practical cases, many of these performance metrics are only credible under presumption of highly rich multipath environments. By highly rich multipath, we mean an environment wherein the random received signals at different ports of the antenna present converged joint probability density functions [3]. Conversely, in actual multipath environments, the number of incoming waves is finite [2]. Under this constraint, diversity performance of a multi-port antenna can be quite different. To take into account the aforementioned issue, the notion of richness threshold has been introduced in [3]. Defined as the minimum number of independent incoming waves required to realize a converged diversity gain, richness threshold plays an important role in multipath environments of finite richness. In general, the lower the richness threshold, the better the multi-port antenna system. Therefore, the best design for a multi-port antenna is the one rendering ideally no correlation, unit total embedded efficiencies, and small richness threshold, i.e., $<5$ waves.

The literature is rich concerning different parameters which affect correlation and total embedded element efficiencies [1], [4]-[10]. Coupling is one of these parameters, which has been well treated. Yet, richness threshold, as a new concept, is almost intact in this respect. Therefore, in the current paper we study the influence of coupling among different elements on this novel performance metric. We will show that high coupling will decrease the richness threshold by increasing the pattern diversity among different antenna elements. In the next section, the simulation approach used in the paper is described. It is followed by study of convergence in the effective diversity gain for different amount of coupling between antenna elements. In section IV, we discuss about convergence in correlations between different antenna elements, which also reveals the role of pattern diversity in the overall performance of multi-port antennas.

\section{Simulation Description}

There are a number of ways to simulate diversity gain among which the frequently used method is the one introduced in [11]. But, a common restriction inherent in almost all of them is that they are most likely only valid for rich multipath environments. Thus, they are not suitable in the frame of current paper. Instead, we use the classic method in which first a multipath environment is emulated wherein the random incoming waves have certain distribution for the angle of arrival (AoA), amplitude and phase and also cross-polarization ratio. Afterwards, the antenna elements are exposed to these incoming waves which superimpose to create different random voltage samples at the ports. In the second round of realization, another random multipath environment with similar features is emulated which, in turn, gives rise to different set of random voltage samples at the ports. This process of realization continues to eventually result in a sufficient number of received random voltage samples [8], [12, ch. 4]. Now, correlations between different pairs of antenna ports can be obtained by calculating cross-correlations between the corresponding received signals $[12, \mathrm{ch} .2]$. Furthermore, to calculate the diversity gain, first we need to find the desired combined signal which depends on the chosen combining scheme (see [13, ch. 7]). Then, we obtain the cumulative density functions (CDF)s of the random received signals plus the combined one. Now, the ratio between the strength of the combined signal and the best received signal at $1 \%$ of their CDFs is the apparent diversity gain (ADG). Limiting ourselves to uniform multipath environments of balanced polarization, the effective diversity gain (EDG), in turn, can be achieved by

$$
\mathrm{EDG}=e_{\max } \mathrm{ADG}
$$

in which $e_{\max }$ is the embedded efficiency of the best antenna element (i.e., the most efficient one). In the frame of this paper, we are concerned about effective diversity gain for maximum 
ratio combining scheme, which is henceforth referred to simply as diversity gain.

In principle, in the simulation approach described above, the distribution of complex electric field of each polarization coming from different AoAs is irrelevant. The reason is, according to the central limit theorem, regardless of the distribution of the incoming waves, as long as they are independent and identically distributed, the distributions of the received complex voltage samples eventually converge to complex Gaussian. The latter is a desirable distribution due to the fact that it gives the maximum diversity gain. That how many incident waves are required to result in converged complex Gaussian distributed random signals at different ports is a question of interest; but, somewhat beyond the scope of this study. To simplify our numerical analysis, let us presumably consider random complex Gaussian incoming waves in our simulations. By this assumption, regardless of the number of incident waves, distribution of the amplitude of random received signals becomes Rayleigh. Under this constraint, convergence in joint probability density function -and thus richness threshold- only depends on convergence in covariance matrix. The latter parameter depends on shape of the embedded far field functions and, therefore, of more interest.

\section{Diversity GAIN STUdY}

There are three different structures upon which we establish our study. The first one is four horizontal parallel dipoles (4HD) at $h=0.15 \lambda_{\circ}\left(\lambda_{\circ}=0.3 m\right)$ height above a perfect electric conductor (PEC) plane. The second structure is four aligned monopoles (4MP) above a PEC, and the last one is six monopoles (6MP) above a PEC plane in a hexagonal configuration. Note that, in all these multi-port radiation structures, the PEC plane extends to infinity. Moreover, the consecutive radiation elements are equidistant and lossless. The resonance frequency of these identical elements in isolated state is around $f_{\circ}=1 \mathrm{GHz}\left(\lambda_{\circ}=0.3 m\right)$.

Let us consider the absolute value of mutual resistance among antenna elements as an indicator of their coupling. In general, for a compact antenna system, the coupling between two consecutive elements is inversely proportional to the distance between them. This means, as the distance between the elements shrinks the total amount of coupling present in the structure increases. Therefore, to study the effects of coupling, we arbitrarily choose different distances between the elements of proposed structures, $d$, referred to as element separation.

Diversity gain of four horizontal dipoles versus the number of incident waves are shown in Fig. 1. Four different curves drawn in the figure are associated with different element separations. The number of realizations used in our simulation is $10^{6}$ rendering a relative error of $\delta< \pm 0.1 \mathrm{~dB}$. Similar simulations are conducted for cases of 4MP and 6MP, whose results are depicted in Figs. $2 \& 3$, respectively.

Note that, in general, coupling affects the overall diversity performance in two ways: by deteriorating the total embedded element efficiencies and through giving rise to correlations.

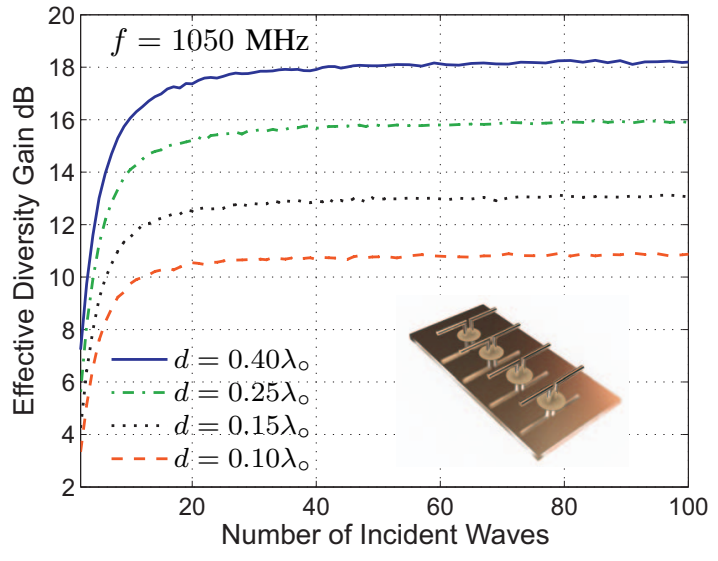

Fig. 1. Diversity gains versus the number of incident waves for four horizontal dipoles (4HD) above a PEC plane.

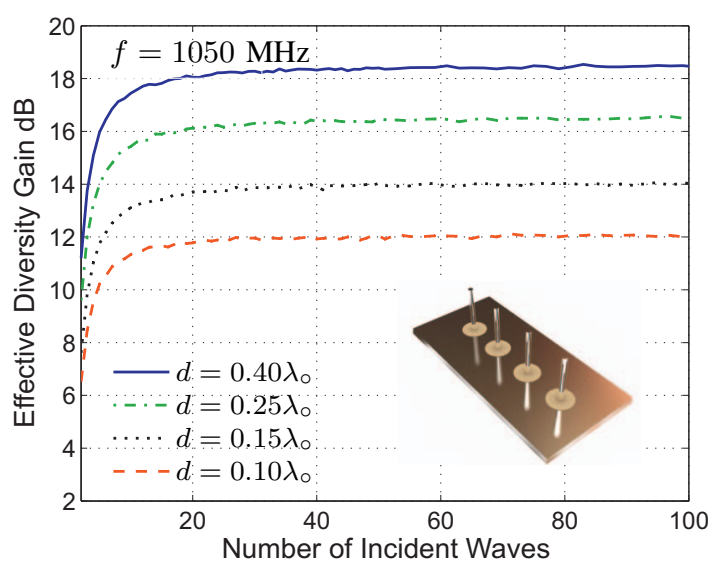

Fig. 2. Diversity gains versus the number of incident waves for four quarter wavelength monopoles (4MP) above a PEC plane.

As the element separation decreases, we expect a reduced diversity gain. However, for the time being, overall reduction of diversity gain performance is of little concern for us. In contrast, the evolution of diversity gains in these figures is more crucial. Thus, for a further comparison let us normalize the diversity gains to their asymptotic values associated with each element separation. Some asymptotic values for 4HD and 4MP antennas with arbitrary element separations are given in Table I. These values are used for normalization in Figs. 4 $\&$ 5. Fig. 4 illustrates the normalized diversity gains for the case of 4HD multi-port antenna. It is clear that with reduced element separation, convergence in diversity gain towards its asymptotic value is faster. Based on this figure, the case of $d=0.05 \lambda_{\circ}$ has the best convergence behavior wherein 15 random incident waves are sufficient for diversity gain to exceed $90 \%(-0.5 \mathrm{~dB})$ of its asymptotic value. In contrast, more than 31 number of independent incident waves are required for element separation of $d=0.40 \lambda_{\circ}$ to overpass the same level.

A similar study on $4 \mathrm{MP}$ and $6 \mathrm{MP}$ antennas reveals that 


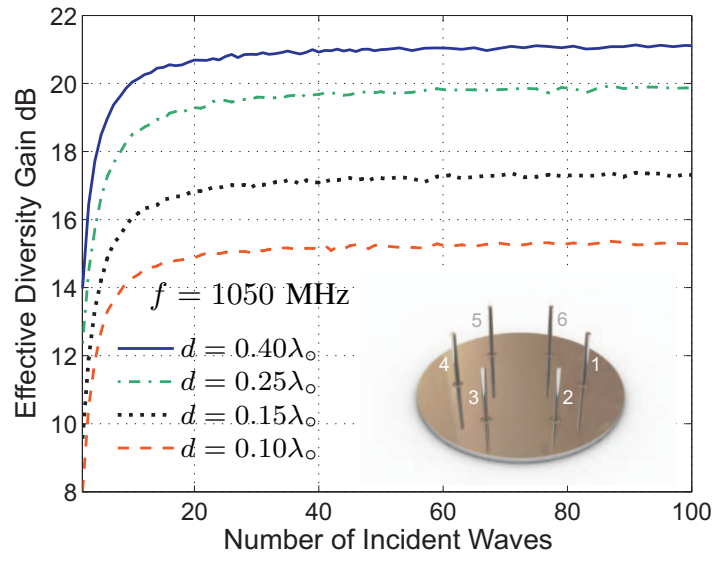

Fig. 3. Diversity gains versus the number of incident waves for six monopoles (6MP) above a PEC plane.

TABLE I

MAXIMUM Diversity GaIN USED TO NoRMALIZE THE RESUltS IN FIGS. 4 \& 5

\begin{tabular}{lrlr}
\hline \multicolumn{4}{c}{ maximum Diversity Gain } \\
\hline Separation & $4 \mathrm{HD}$ & Separation & $4 \mathrm{MP}$ \\
\hline$d=0.05 \lambda_{\circ}$ & $7.9 \mathrm{~dB}$ & $d=0.050 \lambda_{\circ}$ & $9.5 \mathrm{~dB}$ \\
$d=0.10 \lambda_{\circ}$ & $10.9 \mathrm{~dB}$ & $d=0.075 \lambda_{\circ}$ & $10.9 \mathrm{~dB}$ \\
$d=0.15 \lambda_{\circ}$ & $13.1 \mathrm{~dB}$ & $d=0.100 \lambda_{\circ}$ & $12.1 \mathrm{~dB}$ \\
$d=0.25 \lambda_{\circ}$ & $16.0 \mathrm{~dB}$ & $d=0.175 \lambda_{\circ}$ & $14.8 \mathrm{~dB}$ \\
$d=0.40 \lambda_{\circ}$ & $18.3 \mathrm{~dB}$ & $d=0.300 \lambda_{\circ}$ & $17.5 \mathrm{~dB}$ \\
\hline
\end{tabular}

convergence in diversity gain in these two antennas is less affected by the coupling between them. Indeed, for these two cases convergence does not change significantly unless the element separation is very small. Fig. 5 shows the normalized diversity gain for different element separations for the case of 4MP antenna. Should we again consider $90 \%(-0.5 \mathrm{~dB})$ as the threshold level, for $d=0.05 \lambda_{\circ}$ the richness threshold is 13 waves, whereas for $d=0.3 \lambda_{\circ}$ the richness threshold is 22 waves. Table II highlights the threshold richness achieved for some selected element separations for 4HD and 4MP antennas. Note that in this table, the threshold level used to determine the richness threshold is $-0.5 \mathrm{~dB}$ from the corresponding asymptotic values.

A more or less similar result can be achieved for the case of 6MP which is omitted for sake of conciseness. Instead, for this certain case we directly present the richness threshold for different element separation in Fig. 6. It can be inferred that the larger element separation does not necessarily mean the larger richness threshold. Indeed, shape of the embedded far field functions plays the central role in determining the richness threshold. Even so, for highly coupled elements, $d<0.2 \lambda_{\circ}$ the richness threshold reduces consistently.

\section{Convergence in Covariance}

The normalized covariance matrix of received signals at different ports is a square matrix, whose diagonal elements

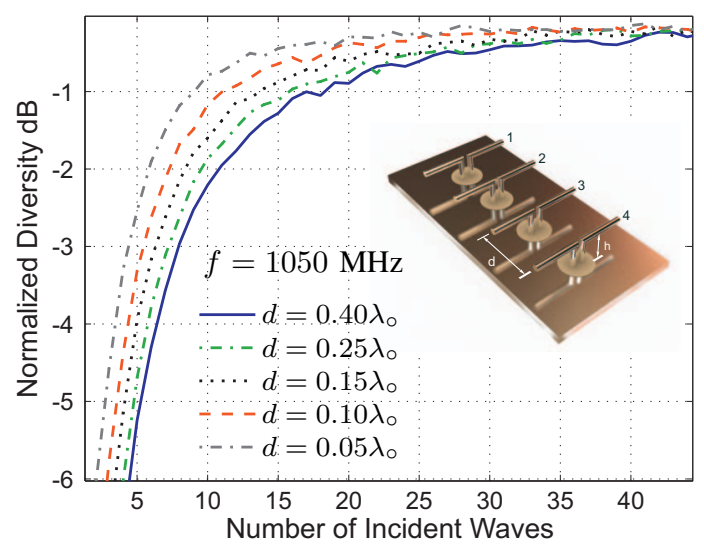

Fig. 4. Normalized diversity gain versus the number of incident waves for four dipoles (4HD) above a PEC plane.

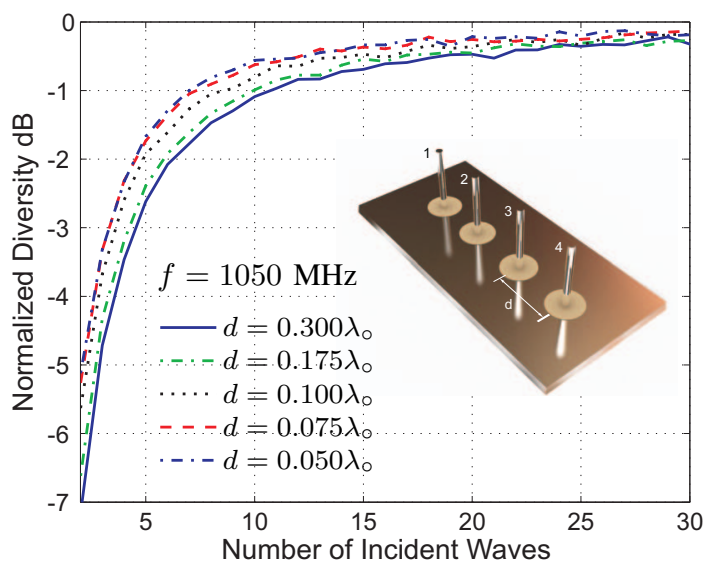

Fig. 5. Normalized diversity gain versus the number of incident waves for four monopoles (4MP) above a PEC plane.

TABLE II

RICHNESS THRESHOLD CALCULATED AT $-0.5 \mathrm{~dB}$ LEVEL FROM ITS ASYMPTOTIC VALUE.

\begin{tabular}{ccccc}
\hline \multicolumn{2}{c}{$4 \mathrm{HD}$} & & \multicolumn{2}{c}{$4 \mathrm{MP}$} \\
\cline { 1 - 2 } \cline { 5 - 5 } dist. & Richness Thr. & & dist. & Richness Thr. \\
\hline$d=0.05 \lambda_{\circ}$ & 15 & & $d=0.050 \lambda_{\circ}$ & 13 \\
$d=0.10 \lambda_{\circ}$ & 19 & & $d=0.075 \lambda_{\circ}$ & 13 \\
$d=0.15 \lambda_{\circ}$ & 26 & & $d=0.100 \lambda_{\circ}$ & 17 \\
$d=0.25 \lambda_{\circ}$ & 27 & & $d=0.175 \lambda_{\circ}$ & 19 \\
$d=0.40 \lambda_{\circ}$ & 31 & & $d=0.300 \lambda_{\circ}$ & 22 \\
\hline
\end{tabular}

are unity whereas off-diagonal elements are the complex correlation coefficients between the received random signals at different antenna ports. Note that the absolute value of complex correlation coefficient is intended here which is henceforth referred to as correlation.

The main goal in this section is to study convergence in covariance matrix versus the number of independent incident waves in a multipath environment. Due to symmetry 


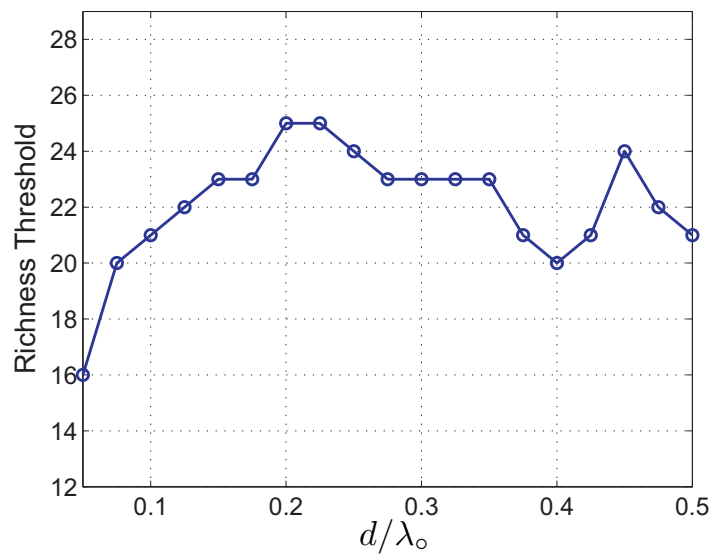

Fig. 6. The $-0.5 \mathrm{~dB}$ richness threshold versus element separation for six monopoles (6MP) above a PEC plane at $f=1050 \mathrm{MHz}$.

of the structure, the covariance matrix of 6MP antenna can be represented only by three different parameters. Thus, we focus on the case of 6MP antenna in this section. If we number the ports of this antenna clockwise, the complete covariance matrix can be represented only by $\rho_{12}, \rho_{13}$, and $\rho_{14}$. Fig. 7 shows the convergence in these correlations versus the number of incident waves. In Fig. 7(a), $\rho_{12}$ for different element separations is depicted. Due to presence of more coupling for shorter separations, $\rho_{12}$ contributes considerably in reduction of diversity gain performance, and is thus of more importance. It is clear from the figure that $\rho_{12}$ for $d=0.10 \lambda_{\circ}$ converges with around 60 number of incident waves, i.e., discrepancy between the corresponding correlation and its asymptotic value is $<0.02$. In contrast, none of the other element separations has a converged $\rho_{12}$ for less than 100 incident waves. Correlations $\rho_{13}$ and $\rho_{14}$ have less contribution in overall diversity performance and as is evident from Figs 7 (b) and 7(c), they do not converge to their asymptotic values even with 100 incident waves.

An important point to remember is that, the asymptotic value of correlations between any pair of ports can also be achieved through the reaction of first embedded far field function with conjugate of the second one [1]. The initial conditions for the aforementioned equality to hold have been expressed as follows: The incoming waves of different AoAs, and the incoming waves of the same AoA but different polarizations be independent. Let us denote the embedded far field function of antenna port $i$, by $\mathbf{G}_{i}=G_{\theta_{i}} \hat{\theta}+G_{\psi_{i}} \hat{\psi}$, and the solid angle in spherical coordinates with $\Omega(\theta, \psi)$. For isotropic multipath, based on the simulation results we can write

$$
\left|\rho_{i j}\right| \geqslant \frac{\left|\oint_{4 \pi} \mathbf{G}_{i}(\Omega) \cdot \mathbf{G}_{j}^{*}(\Omega) d \Omega\right|}{\sqrt{\oint_{4 \pi}\left|\mathbf{G}_{i}(\Omega)\right|^{2} d \Omega \cdot \oint_{4 \pi}\left|\mathbf{G}_{j}(\Omega)\right|^{2} d \Omega}}
$$

The equality occurs when there are a sufficient number of independent incoming waves. In case of single-mode antennas

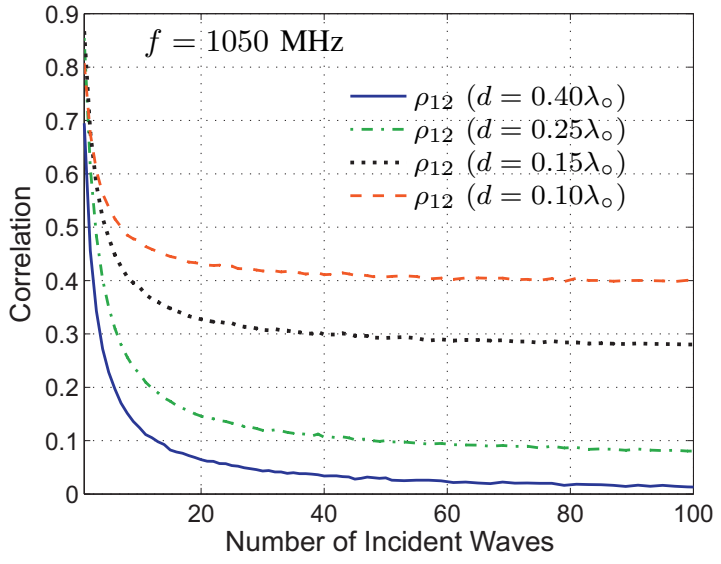

(a) $\rho_{12}$

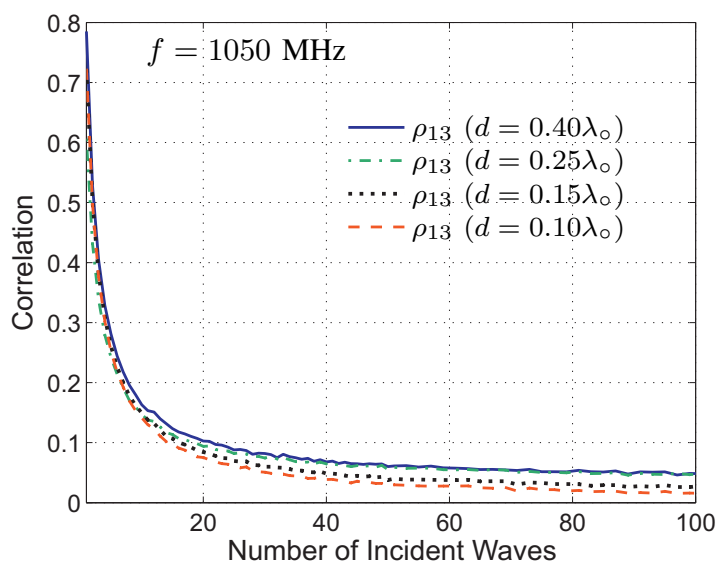

(b) $\rho_{13}$

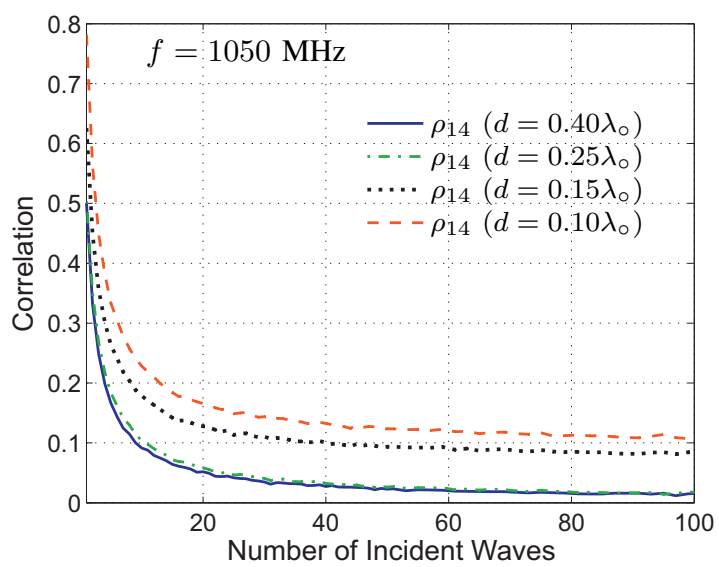

(c) $\rho_{14}$

Fig. 7. Convergence in correlation versus the number of incident waves for six monopoles (6MP) above a PEC plane.

representing minimum scattering antennas, the right hand side of the above equation can also be evaluated by the input network parameters [1]. Table III shows the asymptotic values of correlations for the cases in Fig. 7 obtained by the 
TABLE III

Converged Correlation for 6MP shown in Fig. 7 AChieved by $\sqrt{\rho_{e}}$ Given In [14, EQ. 1$]$.

\begin{tabular}{ccccc}
\hline Correlation & $d=0.10 \lambda_{\circ}$ & $d=0.15 \lambda_{\circ}$ & $d=0.25 \lambda_{\circ}$ & $d=0.40 \lambda_{\circ}$ \\
\hline$\rho_{12}$ & 0.40 & 0.27 & 0.07 & 0.00 \\
$\rho_{13}$ & 0.00 & 0.01 & 0.03 & 0.03 \\
$\rho_{14}$ & 0.10 & 0.07 & 0.01 & 0.01 \\
\hline
\end{tabular}

corresponding input network parameters. In general, as the number of incident waves reduces, the correlation increases monotonically. These simulations clarify that there is an upper bound for each correlation which is not necessarily 1 . For instance, an interesting case happens when there is only a single random incident wave. In this particular case, if AoA in each realization were fixed, correlations between any pair of received signals would be one. However, in the current simulations, for each realization the AoA changes randomly. Let us further denote correlation between the received signals at ports $i$ and $j$ for a single random incoming wave by $\varrho_{i j}$. This parameter is a measure of dissimilarities between the two embedded far field functions regardless of the distance between their phase centers. Indeed, it quantifies what is known among antenna engineers as pattern diversity. For instance, if $\varrho_{i j}=0$ (i.e., no correlation), we have $100 \%$ pattern diversity and if $\varrho_{i j}=1$ (i.e., fully correlated signals), there exists no pattern diversity.

$$
1 \geqslant\left|\varrho_{i j}\right| \geqslant\left|\rho_{i j}\right|
$$

\section{CONCLUSION}

In this paper, richness threshold for different element separations in some proposed multi-port antennas has been studied. The results reveal that highly coupled elements present faster convergence in diversity gain and therefore lower richness threshold. This convergence can be attributed to the pattern diversity which is caused by strong coupling among different elements. We conclude that diversity gain convergence -and thus the richness threshold- is in general a function of shapes of the embedded far field functions. Furthermore, we have linked the convergence in diversity gain to convergence in correlations at the ports of the multi-port antennas. It is shown that there is a great deal of parity between these two parameters. The concept of pattern diversity, which is well-known among antenna engineers, has been quantified in this paper. By simulations, we have shown that in an ideal uncorrelated multipath environment, the spatial correlation at different ports varies between the pattern diversity measure and the prevalent expression in (1) well-known in the literature. We stress that the latter is in general valid in a rich multipath environment wherein the number of independent incident waves is sufficiently large. But, the actual value of this spatial correlation is a function of the number of these incoming waves.

\section{ACKNOWLEDGMENT}

The computations were performed on C3SE computing resources in Gothenburg, Sweden.

\section{REFERENCES}

[1] R. Vaughan and J. Andersen, "Antenna diversity in mobile communications," IEEE Transactions on Vehicular Technology, vol. 36, no. 4, pp. 149-172, November 1987.

[2] T. Taga, "Analysis for mean effective gain of mobile antennas in land mobile radio environments," IEEE Transactions on Vehicular Technology, vol. 39, no. 2, pp. 117-131, May 1990.

[3] N. Jamaly, M. T. Iftikhar, and Y. Rahmat-Samii, "Performance evaluation of diversity antennas in multipath environments of finite richness," in 2012 Proceedings of the Sixth European Conference on Antennas and Propagation (EuCAP), March 2012.

[4] A. Derneryd and G. Kristensson, "Antenna signal correlation and its relation to the impedance matrix," Electronics Letters, vol. 40, no. 7, pp. 401-402, April 2004.

[5] J. W. Wallace and M. A. Jensen, "Termination-dependent diversity performance of coupled antennas: Network theory analysis," IEEE Transactions on Antennas and Propagation, vol. 52, no. 1, pp. 98-105, January 2004.

[6] M. A. Jensen and J. W. Wallace, "A review of antennas and propagation for MIMO wireless communications," IEEE Transactions on Antennas and Propagation, vol. 52, no. 11, pp. 2810-2824, November 2004.

[7] M. Özdemir, E. Arvas, and H. Arslan, "Dynamics of spatial correlation and implications on MIMO systems," IEEE Communications Magazine, vol. 42, no. 6, pp. S14-S19, June 2004.

[8] K. Rosengren and P.-S. Kildal, "Radiation efficiency, correlation, diversity gain and capacity of six monopole antenna array for a MIMO system: Theory, simulation and measurement in reverberation chamber," Proceedings IEE, Microwaves Antennas and Propagation, 2005.

[9] N. Jamaly, C. Gomez-Calero, P.-S. Kildal, J. Carlsson, and A. Wolfgang, "Study of excitation on beam ports versus element ports in performance evaluation of diversity and MIMO arrays," in 2009 Proceedings of the Third European Conference on Antennas and Propagation (EuCAP), March 2009.

[10] C. Waldschmidt, S. Schulteis, and W. Wiesbeck, "Complete RF system model for analysis of compact MIMO arrays," IEEE Transactions on Vehicular Technology, vol. 53, no. 3, pp. 579-586, May 2004.

[11] O. Norklit, P. Teal, and R. Vaughan, "Measurement and evaluation of multi-antenna handsets in indoor mobile communication," IEEE Transactions on Antennas and Propagation, vol. 49, no. 3, pp. 429437, March 2001.

[12] N. Jamaly, "Spatial characterization of multi-element antennas," Chalmers University of Technology, available online at http://publications.lib.chalmers.se/records/fulltext/137635.pdf, Tech. Rep. R002/2011, 2011.

[13] A. Goldsmith, Wireless Communications. Cambridge University Press, 2005.

[14] S. Blanch, J. Romeu, and I. Corbella, "Exact representation of antenna system diversity performance from input parameter description," Electronics Letters, vol. 39, no. 9, pp. 705-707, May 2003. 